\title{
Dynamics Analysis of a Viral Infection Model with a General Standard Incidence Rate
}

\author{
Yu Ji and Muxuan Zheng \\ Department of Mathematics, Beijing Technology and Business University, Beijing 100048, China \\ Correspondence should be addressed to Yu Ji; jyslt@163.com
}

Received 23 May 2014; Accepted 20 August 2014; Published 14 October 2014

Academic Editor: Peixuan Weng

Copyright ( 2014 Y. Ji and M. Zheng. This is an open access article distributed under the Creative Commons Attribution License, which permits unrestricted use, distribution, and reproduction in any medium, provided the original work is properly cited.

The basic viral infection models, proposed by Nowak et al. and Perelson et al., respectively, have been widely used to describe viral infection such as HBV and HIV infection. However, the basic reproduction numbers of the two models are proportional to the number of total cells of the host's organ prior to the infection, which seems not to be reasonable. In this paper, we formulate an amended model with a general standard incidence rate. The basic reproduction number of the amended model is independent of total cells of the host's organ. When the basic reproduction number $R_{0}<1$, the infection-free equilibrium is globally asymptotically stable and the virus is cleared. Moreover, if $R_{0}>1$, then the endemic equilibrium is globally asymptotically stable and the virus persists in the host.

\section{Introduction}

Mathematical models of viral infection have played a significant role in the understanding of the disease in vivo [1]. Analysis of the viral dynamics of a proper model can not only provide important quantitative insights into the pathogenesis, but also lead to design treatment strategies which would more effectively bring the infection under control [2].

The basic models of within-host viral infection, proposed by Nowak and May [3] and Perelson and Nelson [4], have been widely used in the studies of viral infection [5-13], such as HBV and HIV infection. In both of these two basic models, uninfected cells $x$ are assumed to become infected by free virions $v$ at the bilinear rate $\beta x v$, where $\beta$ is a positive constant rate. However, the basic reproduction number $R_{0}$ of these two models is proportional to the number of total cells of the host's organ prior to the infection. This implies that an individual with a smaller organ maybe more resistant to virus infection than an individual with a larger one. Hence, Min et al. [5] proposed the following amended Nowak and May's model with a standard incidence rate to describe the hepatitis $B$ virus infection:

$$
\begin{aligned}
& x^{\prime}=\lambda-d x-\beta \frac{x}{x+y} v, \\
& y^{\prime}=\beta \frac{x}{x+y} v-a y, \\
& v^{\prime}=k y-u v .
\end{aligned}
$$

The basic reproduction number $R_{0}$ of model (1) is independent of the number of total cells of the host's organ.

In the modelling the viral infection of disease, the incidence rate, which is the rate of new infections, plays an important role in describing the viral dynamics. Bilinear and standard incidence rate are the most common incidence rates in virus infection models. However, there are still some other nonlinear incidence rates to describe disease infections. Yorke and London [14] investigated an incidence rate $x g(v)=$ $\beta x v(1-c v)$ for measles outbreaks. Liu et al. [15] studied a nonlinear saturated mass action given by $\beta x\left(v^{p} /\left(1+\alpha v^{q}\right)\right)$, where $\beta, p, \alpha$, and $q>0$. When $p=q=1$, the nonlinear incidence rate becomes $\beta x(v /(1+\alpha v))$, which has been frequently used in the viral model with saturation response. 
In this paper, motivated by the above models, we formulate an amended viral infection model with a general standard incidence rate, which is described as follows:

$$
\begin{aligned}
& x^{\prime}=\lambda-d x-\beta \frac{x}{x+y} g(v), \\
& y^{\prime}=\beta \frac{x}{x+y} g(v)-a y, \\
& v^{\prime}=k y-u v,
\end{aligned}
$$

where $g(0)=0, g^{\prime}(v)>0$, and $g^{\prime \prime}(v) \leqslant 0$ when $v \geqslant 0$. Under this assumption, in the special case $g(v)=v$, the incidence rate means the standard incidence rate. If $g(v)=v /(1+\alpha v)$, then that describes the model with the standard incidence rate and saturation response.

The basic reproduction number of the model (2) is given by $R_{0}=(\beta k / a u) g^{\prime}(0)$, which describes the average number of secondary infections produced by a single infected cell during the period of infection when all cells are uninfected. Clearly, $R_{0}$ of model (2) is also independent of the number of the host's organ. The main purpose of this paper is to study the virus dynamics of model (2).

The rest of this paper is organized as follows: Section 2 studies the existence and uniqueness of equilibria of model (2). The stability of the infection-free equilibrium and the endemic equilibrium is analyzed in Section 3. Finally, concluding remarks are given in Section 4.

\section{The Existence and Uniqueness of Equilibria}

Before the analysis of the existence and uniqueness of equilibria, we will show the positivity and boundedness of solutions of model (2).

2.1. Positivity and Boundedness. The proof of positive solution is easy; we only show the boundedness of solution in the following.

Theorem 1. There is an $M>0$, such that, for any positive solution $(x(t), y(t), v(t))$ of model (2), one has $x(t)<M$, $y(t)<M, v(t)<M$.

Proof. Let

$$
V_{1}(t)=x(t)+y(t)+\frac{a}{2 k} v(t)
$$

Calculating the derivative of $V_{1}$ along the solutions of model (2) gives

$$
\begin{aligned}
V_{1}^{\prime}(t) & =\lambda-d x-\frac{a}{2} y-\frac{a u}{2 k} v \\
& \leqslant \lambda-\min \left\{d, \frac{a}{2}, u\right\} V_{1}(t)
\end{aligned}
$$

Denote $h=\min \{d, a / 2, u\}$; it follows that

$$
V_{1}^{\prime}(t) \leqslant \lambda-h V_{1}(t)
$$

Further

$$
V_{1}(t) \leqslant \frac{\lambda}{h}+\left(V_{1}(0)-\frac{\lambda}{h}\right) e^{-h t} .
$$

Hence, $V_{1}(t)$ is bounded. Then we can conclude that $x(t), y(t)$, and $v(t)$ are eventually bounded. Thus, there exists an $M>0$ such that $x(t)<M, y(t)<M, v(t)<M$. This completes the proof.

Define

$$
\begin{gathered}
D=\left\{(x, y, v) \in R_{+}^{3} \mid 0<x(t) \leqslant \frac{\lambda}{d},\right. \\
0 \leqslant y(t), v(t) \leqslant M\} .
\end{gathered}
$$

If $x(0) \leqslant \lambda / d$, from the first equation of model (2), we have $x(t) \leqslant \lambda / d$ when $t>0$. It is easy to see that $D$ is a positively invariant region for model (2).

2.2. Existence and Uniqueness of the Endemic Equilibrium. Obviously, $Q_{1}=(\lambda / d, 0,0)$ is the infection-free equilibrium of model (2), which represents the extinction of the free virus. As for the existence and uniqueness of the positive equilibrium, we have the following theorem.

Theorem 2. If $R_{0}>1$, then the model (2) has a unique endemic equilibrium of the form $Q_{2}=\left(x^{*}, y^{*}, v^{*}\right)$ with $0<$ $x^{*}<\lambda / d, y^{*}>0$ and $v^{*}>0$.

Proof. At any equilibrium, the following equations hold:

$$
\begin{gathered}
\lambda-d x-\frac{\beta x}{x+y} g(v)=0, \\
\frac{\beta x}{x+y} g(v)-a y=0, \\
k y-u v=0 .
\end{gathered}
$$

By the first and the second equations of (8), we have $y=$ $(1 / a)(\lambda-d x)$. From the third equation, we get $v=(k / u) y$.

Now, we consider the following function $F(x)$ defined on the interval $[0, \lambda / d]$ :

$$
F(x)=\beta x \frac{1}{x+y} g(v)-a y,
$$

$$
\text { where } y=\frac{1}{a}(\lambda-d x), \quad v=\frac{k}{u} y \text {. }
$$

Therefore

$$
\begin{aligned}
F^{\prime}(x)= & \beta \frac{1}{x+y} g(v)-\beta x g(v) \frac{1}{(x+y)^{2}}\left(1-\frac{d}{a}\right) \\
& +\frac{\beta x}{x+y} g^{\prime}(v) \frac{k}{u}\left(-\frac{d}{a}\right)-a\left(-\frac{d}{a}\right) \\
= & \frac{\beta g(v)}{x+y}-\frac{\beta x g(v)}{(x+y)^{2}}+\frac{d}{a} \frac{\beta x g(v)}{(x+y)^{2}}
\end{aligned}
$$




$$
\begin{aligned}
& +d-d \frac{x}{x+y} \frac{\beta k}{a u} g^{\prime}(v) \\
& =\frac{\beta y g(v)}{(x+y)^{2}}+\frac{d}{a} \frac{\beta x g(v)}{(x+y)^{2}}+d \\
& -d \frac{x}{x+y} \frac{\beta k}{a u} g^{\prime}(v) .
\end{aligned}
$$

Clearly, $F(0)=-\lambda<0, F(\lambda / d)=\beta g(0)=0$. Notice that $F^{\prime}(\lambda / d)=d-d(\beta k / a u) g^{\prime}(0)=d\left(1-R_{0}\right)$. Hence, we get $F^{\prime}(\lambda / d)<0$ when $R_{0}>1$. Therefore, there exists at least one positive equilibrium $Q_{2}=\left(x^{*}, y^{*}, v^{*}\right)$ with $0<x^{*}<$ $\lambda / d, y^{*}>0$ and $v^{*}>0$.

Next, we will proof the uniqueness of the endemic equilibrium. Observe the terms $d-d\left(x^{*} /\left(x^{*}+\right.\right.$ $\left.\left.y^{*}\right)\right)(\beta k / a u) g^{\prime}\left(v^{*}\right)$ in $F^{\prime}\left(x^{*}\right)$. Since $x^{*} /\left(x^{*}+y^{*}\right)=$ $a y^{*} / \beta g\left(v^{*}\right), y^{*}=(u / k) v^{*}$ at any positive equilibrium from (8), we have

$$
\begin{aligned}
d- & d \frac{x^{*}}{x^{*}+y^{*}} \frac{\beta k}{a u} g^{\prime}\left(v^{*}\right) \\
& =d-d \frac{a u}{\beta k} \frac{v^{*}}{g\left(v^{*}\right)} \frac{\beta k}{a u} g^{\prime}\left(v^{*}\right) \\
& =d g\left(v^{*}\right)\left(g\left(v^{*}\right)-v^{*} g^{\prime}\left(v^{*}\right)\right) \\
& =d g\left(v^{*}\right)\left(g\left(v^{*}\right)-g(0)-v^{*} g^{\prime}\left(v^{*}\right)\right) \\
& =d g\left(v^{*}\right)\left(v^{*} g^{\prime}(\xi)-v^{*} g^{\prime}\left(v^{*}\right)\right) \\
& =d v^{*} g\left(v^{*}\right)\left(g^{\prime}(\xi)-g^{\prime}\left(v^{*}\right)\right), \\
& \text { where } \xi \in\left(0, v^{*}\right) .
\end{aligned}
$$

Since $g^{\prime \prime} \leqslant 0$, we get $d-d\left(x^{*} /\left(x^{*}+y^{*}\right)\right)(\beta k / a u) g^{\prime}\left(v^{*}\right) \geqslant 0$; that is

$$
a u-\beta k \frac{x^{*}}{x^{*}+y^{*}} g^{\prime}\left(v^{*}\right) \geqslant 0 \text {. }
$$

Consequently, we deduce that $F^{\prime}\left(x^{*}\right)>0$ at any positive equilibrium. Suppose there are at least two positive equilibria of $F(x)=0$ in $(0, \lambda / d)$; then there must be $F^{\prime}\left(x^{*}\right)<0$ at some equilibrium, which is a contradiction. Therefore, if $R_{0}>1$, there exists a unique endemic equilibrium $Q_{2}=\left(x^{*}, y^{*}, v^{*}\right)$ with $0<x^{*}<\lambda / d, y^{*}>0$ and $v^{*}>0$. This completes the proof.

\section{Stability Analysis of Equilibria}

In this section, we will analyze the stability of those two steady states. The Jacobian matrix of the vector field corresponding to model (2) is

$$
J=\left(\begin{array}{ccc}
-d-\frac{\beta y g(v)}{(x+y)^{2}} & \frac{\beta x g(v)}{(x+y)^{2}} & -\frac{\beta x g^{\prime}(v)}{x+y} \\
\frac{\beta y g(v)}{(x+y)^{2}} & -a-\frac{\beta x g(v)}{(x+y)^{2}} & \frac{\beta x g^{\prime}(v)}{x+y} \\
0 & k & -u
\end{array}\right) .
$$

3.1. Stability of the Infection-Free Equilibrium $Q_{1}$. First of all, we will study the stability of the infection-free equilibrium $Q_{1}$.

Theorem 3. If $R_{0}<1$, then $Q_{1}$ is globally asymptotically stable and becomes unstable when $R_{0}>1$.

Proof. For $Q_{1}=(\lambda / d, 0,0)$, the characteristic equation of (13) is given by

$$
\left|s E-J\left(Q_{1}\right)\right|=(s+d)\left(s^{2}+(a+u) s+a u-\beta k g^{\prime}(0)\right)=0 \text {. }
$$

Note that $d>0, a+u>0$ and $a u-\beta k g^{\prime}(0)=a u\left(1-R_{0}\right)>0$ when $R_{0}<1$; thus all eigenvalues of (14) have negative real parts if $R_{0}<1$ and at least one eigenvalue becomes positive when $R_{0}>1$. Hence, $Q_{1}$ is locally asymptotically stable if $R_{0}<1$ and becomes unstable if $R_{0}>1$.

Consider the Lyapunov function

$$
V_{2}=y(t)+\frac{a}{k} v(t) .
$$

Calculating the derivative of $V_{2}$ along the solutions of the model (2) gives

$$
\begin{aligned}
V_{2}^{\prime}(t) & =\frac{\beta x g(v)}{x+y}-\frac{a u}{k} v \\
& \leqslant \beta g(v)-\frac{a u}{k} v \\
& =\beta(g(v)-g(0))-\frac{a u}{k} v \\
& =\beta v g^{\prime}(\xi)-\frac{a u}{k} v \\
& \leqslant \beta v g^{\prime}(0)-\frac{a u}{k} v \\
& =\frac{a u}{k} v\left(R_{0}-1\right)
\end{aligned}
$$

Since $R_{0}<1$, then $V_{2}^{\prime}(t) \leqslant 0$ and $V_{2}^{\prime}(t)=0$ only if $v=0$. By the Lyapunov-Lasalle Theorem, solutions in $D$ approach the largest positively invariant subset of the set $E$ where $V_{2}^{\prime}(t)=$ 0 . Thus, all solutions in the set $E$ approach the infection-free equilibrium $Q_{1}$. This completes the proof. 
3.2. Stability of the Endemic Equilibrium $Q_{2}$. When $R_{0}>$ 1 , there physically exists another steady state $Q_{2}$. We now consider the local stability of the endemic steady state $Q_{2}=$ $\left(x^{*}, y^{*}, v^{*}\right)$ when $R_{0}>1$.

Theorem 4. If $R_{0}>1$, then $Q_{2}$ is locally asymptotically stable.

Proof. The Jacobian matrix of (13) at $Q_{2}$ becomes

$$
J\left(Q_{2}\right)=\left(\begin{array}{ccc}
-d-\frac{\beta y^{*} g\left(v^{*}\right)}{\left(x^{*}+y^{*}\right)^{2}} & \frac{\beta x^{*} g\left(v^{*}\right)}{\left(x^{*}+y^{*}\right)^{2}} & -\frac{\beta x^{*} g^{\prime}\left(v^{*}\right)}{x^{*}+y^{*}} \\
\frac{\beta y^{*} g\left(v^{*}\right)}{\left(x^{*}+y^{*}\right)^{2}} & -a-\frac{\beta x^{*} g\left(v^{*}\right)}{\left(x^{*}+y^{*}\right)^{2}} & \frac{\beta x^{*} g^{\prime}\left(v^{*}\right)}{x^{*}+y^{*}} \\
0 & k & -u
\end{array}\right) .
$$

The characteristic equation associated with $J\left(Q_{2}\right)$ is given by

$$
\left|s E-J\left(Q_{2}\right)\right|=s^{3}+a_{1} s^{2}+a_{2} s+a_{3}=0,
$$

where

$$
\begin{aligned}
a_{1}= & a+u+d+\frac{\beta y^{*} g\left(v^{*}\right)}{\left(x^{*}+y^{*}\right)^{2}}+\frac{\beta x^{*} g\left(v^{*}\right)}{\left(x^{*}+y^{*}\right)^{2}}, \\
a_{2}= & u d+a d+u \frac{\beta g\left(v^{*}\right)}{x^{*}+y^{*}}+\frac{\beta a y^{*} g\left(v^{*}\right)}{\left(x^{*}+y^{*}\right)^{2}} \\
& +\left(a u-\frac{\beta k x^{*} g^{\prime}\left(v^{*}\right)}{x^{*}+y^{*}}\right) \\
a_{3}= & u d \cdot \frac{\beta x^{*} g\left(v^{*}\right)}{\left(x^{*}+y^{*}\right)^{2}+u \cdot \frac{\beta a y^{*} g\left(v^{*}\right)}{\left(x^{*}+y^{*}\right)^{2}}} \\
& +d \cdot\left(a u-\frac{\beta k x^{*} g^{\prime}\left(v^{*}\right)}{x^{*}+y^{*}}\right) .
\end{aligned}
$$

From the inequality of (12), we get $a_{1}>0, a_{2}>0, a_{3}>0$. By Routh-Hurwitz criterion, we are only to show $a_{1} a_{2}>a_{3}$. In fact, all the terms of $a_{1}, a_{2}$, and $a_{3}$ are nonnegative, and all the three terms in $a_{3}$ appear in the expansion of $a_{1} a_{2}$. Hence, $Q_{2}$ is locally asymptotically stable when $R_{0}>1$.

Furthermore, to analyze the global asymptotic stability of $Q_{2}$, we introduce the results of Theorem 2.5 in [16].

Lemma 5. Assume that

(1) there exists a compact absorbing set $K \subset D$ and the system (2) has a unique equilibrium $\bar{x}$ in $D$;

(2) the system (2) satisfies the Poincaré-Bendixson property;

(3) For each periodic solution $x=p(t)$ to (2) with $p(0) \in D$, the second additive compound matrix of the Jacobian (17) is asymptotically stable;

(4) $(-1)^{n} \operatorname{det}((\partial f / \partial x)(\bar{x}))>0$.

Then the unique equilibrium $\bar{x}$ is globally asymptotically stable in $D$.
Therefore, we only need to prove that model (2) at the $Q_{2}$ satisfies this lemma when $R_{0}>1$. Then we have the following conclusion.

Theorem 6. If $R_{0}>1$, then $Q_{2}$ is globally asymptotically stable.

Proof. Firstly, the assumption (1) of Lemma 5 is equivalent to the uniform persistence of the model (2) [17]. Clearly, the $Q_{1}$ is a unique steady state at the boundary of $D$. And the uniform persistence of (2) is equivalent to the instability of the $Q_{1}$ [18]. Since the infected-free steady state $Q_{1}$ is unstable when $R_{0}>$ 1, the assumption (1) of Lemma 5 holds.

Secondly, in order to verify assumption (2) of Lemma 5, we only need to show that model (2) is competitive in the convex region $D$. Taking the diagonal matrix $H=$ $\operatorname{diag}(1,-1,1)$, it is easy to verify that the matrix $H J H$ has nonpositive off-diagonal elements, where $J$ is the Jacobian matrix (17). Hence, model (2) is competitive. So model (2) satisfies the Poincaré-Bendixson property.

Next, we will show that model (2) satisfies assumption (3) of Lemma 5. The second additive compound matrix of the Jacobian matrix (13) is

$$
J^{[2]}=\left(\begin{array}{ccc}
-d-a-\frac{\beta g(v)}{x+y} & \frac{\beta x g^{\prime}(v)}{x+y} & \frac{\beta x g^{\prime}(v)}{x+y} \\
k & -d-u-\frac{\beta y g(v)}{x+y} & \frac{\beta x g(v)}{(x+y)^{2}} \\
0 & \frac{\beta y g(v)}{(x+y)^{2}} & -u-a-\frac{\beta x g(v)}{(x+y)^{2}}
\end{array}\right) .
$$

The second compound system (2) along a periodic solution $p(t)=(x(t), y(t), v(t))$ is

$$
\begin{aligned}
& W_{1}^{\prime}=\left(-d-a-\frac{\beta g(v)}{x+y}\right) W_{1}+\frac{\beta x g^{\prime}(v)}{x+y}\left(W_{2}+W_{3}\right), \\
& W_{2}^{\prime}=k W_{1}-\left(d+u+\frac{\beta y g(v)}{x+y}\right) W_{2}+\frac{\beta x g(v)}{(x+y)^{2}} W_{3}, \\
& W_{3}^{\prime}=\frac{\beta y g(v)}{(x+y)^{2}} W_{2}-\left(u+a+\frac{\beta x g(v)}{(x+y)^{2}}\right) W_{3} .
\end{aligned}
$$

To show that the model (21) is asymptotically stable, consider a Lyapunov function

$$
V\left(W_{1}, W_{2}, W_{3}, p\right)=\sup \left\{\left|W_{1}\right|, \frac{y(t)}{v(t)}\left(\left|W_{2}\right|+\left|W_{3}\right|\right)\right\} .
$$

From the uniform persistence, there exists a constant $\eta>0$ such that

$$
V \geqslant \eta \cdot \sup \left\{\left|W_{1}\right|,\left|W_{2}\right|,\left|W_{3}\right|\right\}
$$


The right derivative of $V$ along a solution $\left(W_{1}, W_{2}, W_{3}\right)$ to (21) and $(x(t), y(t), v(t))$ can be estimated as follows:

$$
\begin{aligned}
D_{+}\left|W_{1}\right| \leqslant & \left(-d-a-\frac{\beta g(v)}{x+y}\right)\left|W_{1}\right| \\
& +\frac{\beta x g^{\prime}(v)}{x+y}\left(\left|W_{2}\right|+\left|W_{3}\right|\right), \\
D_{+}\left|W_{2}\right| \leqslant & k\left|W_{1}\right|-\left(d+u+\frac{\beta y g(v)}{x+y}\right)\left|W_{2}\right| \\
& +\frac{\beta x g(v)}{(x+y)^{2}}\left|W_{3}\right|, \\
D_{+}\left|W_{3}\right| \leqslant & \frac{\beta y g(v)}{(x+y)^{2}}\left|W_{2}\right|-\left(u+a+\frac{\beta x g(v)}{(x+y)^{2}}\right)\left|W_{3}\right| .
\end{aligned}
$$

Then, we will get

$$
\begin{aligned}
D_{+} & \frac{y(t)}{v(t)}\left(\left|W_{2}\right|+\left|W_{3}\right|\right) \\
= & \left(\frac{y^{\prime}(t)}{y(t)}-\frac{v^{\prime}(t)}{v(t)}\right) \frac{y(t)}{v(t)}\left(\left|W_{2}\right|+\left|W_{3}\right|\right) \\
& +\frac{y(t)}{v(t)} D_{+}\left(\left|W_{2}\right|+\left|W_{3}\right|\right) \\
\leqslant & k \frac{y}{v}\left|W_{1}\right|+\frac{y}{v}\left(\frac{y^{\prime}}{y}-\frac{v^{\prime}}{v}-u-\min (a, d)\right) \\
& \times\left(\left|W_{2}\right|+\left|W_{3}\right|\right) .
\end{aligned}
$$

Therefore,

$$
D_{+} V(t) \leqslant \sup \left(g_{1}(t), g_{2}(t)\right) V(t)
$$

where

$$
\begin{aligned}
g_{1}(t)= & -d-a-\frac{\beta g(v)}{x+y}+\frac{v}{y} \frac{\beta x}{x+y} g^{\prime}(v) \\
\leqslant & -d-a+\frac{1}{y} \frac{\beta x}{x+y} v g^{\prime}(v), \\
g_{2}(t)= & k \frac{y}{v}+\frac{y^{\prime}}{y}-\frac{v^{\prime}}{v}-u \\
& -\min (a, d)=\frac{y^{\prime}}{y}-\min (a, d) .
\end{aligned}
$$

Notice that $g^{\prime \prime}<0, g(v)=g(v)-g(0)=v g^{\prime}(\xi) \geqslant v g^{\prime}(v)$, where $\xi \in(0, v)$. We get

$$
\begin{aligned}
g_{1}(t) & \leqslant-d-a+\frac{1}{y} \frac{\beta x}{x+y} g(v) \\
& =-d-a+\frac{1}{y}\left(y^{\prime}+a y\right)=-d+\frac{y^{\prime}}{y} .
\end{aligned}
$$

Denote $h=\min (a, d)$; we get

$$
\sup \left(g_{1}(t), g_{2}(t)\right) \leqslant \frac{y^{\prime}}{y}-h \text {. }
$$

By inequality (26) and Gronwall inequality, we have

$$
V(t) \leqslant V(0) y(t) e^{-h t} \leqslant V(0) M e^{-h t}
$$

This implies that $V(0) \rightarrow 0$ when $t \rightarrow+\infty$. And from inequality (23), we get $\left(W_{1}(t), W_{2}(t), W_{3}(t)\right) \rightarrow 0$ when $t \rightarrow$ $+\infty$. As a result, the second additive compound matrix of the Jacobian (17) is asymptotically stable.

At last, we will prove that model (2) satisfies assumption (4) of Lemma 5. Let $J\left(Q_{2}\right)$ be the Jacobian matrix of (2) at $Q_{2}$. Then

$$
\begin{aligned}
& \operatorname{det}\left(J\left(Q_{2}\right)\right) \\
& =\left|\begin{array}{ccc}
-d-\frac{\beta y^{*} g\left(v^{*}\right)}{\left(x^{*}+y^{*}\right)^{2}} & \frac{\beta x^{*} g\left(v^{*}\right)}{\left(x^{*}+y^{*}\right)^{2}} & -\frac{\beta x^{*} g^{\prime}\left(v^{*}\right)}{x^{*}+y^{*}} \\
\frac{\beta y^{*} g\left(v^{*}\right)}{\left(x^{*}+y^{*}\right)^{2}} & -a-\frac{\beta x^{*} g\left(v^{*}\right)}{\left(x^{*}+y^{*}\right)^{2}} & \frac{\beta x^{*} g^{\prime}\left(v^{*}\right)}{x^{*}+y^{*}} \\
0 & k & -u
\end{array}\right| \\
& =-u\left(d+\frac{\beta y^{*} g\left(v^{*}\right)}{\left(x^{*}+y^{*}\right)^{2}}\right)\left(a+\frac{\beta x^{*} g\left(v^{*}\right)}{\left(x^{*}+y^{*}\right)^{2}}\right) \\
& -k \frac{\beta y^{*} g\left(v^{*}\right)}{\left(x^{*}+y^{*}\right)^{2}} \cdot \frac{\beta x^{*} g^{\prime}\left(v^{*}\right)}{x^{*}+y^{*}} \\
& +u \frac{\beta x^{*} g\left(v^{*}\right)}{\left(x^{*}+y^{*}\right)^{2}} \frac{\beta y^{*} g\left(v^{*}\right)}{\left(x^{*}+y^{*}\right)^{2}} \\
& +k \frac{\beta x^{*} g^{\prime}\left(v^{*}\right)}{x^{*}+y^{*}} \cdot\left(d+\frac{\beta y^{*} g\left(v^{*}\right)}{\left(x^{*}+y^{*}\right)^{2}}\right) \\
& =- \text { aud }-u d \frac{\beta x^{*} g\left(v^{*}\right)}{\left(x^{*}+y^{*}\right)^{2}} \\
& -a u \frac{\beta y^{*} g\left(v^{*}\right)}{\left(x^{*}+y^{*}\right)^{2}}+k d \frac{\beta x^{*} g^{\prime}\left(v^{*}\right)}{x^{*}+y^{*}} \\
& =-d\left(a u-k \frac{\beta x^{*} g^{\prime}\left(v^{*}\right)}{x^{*}+y^{*}}\right)-u d \frac{\beta x^{*} g\left(v^{*}\right)}{\left(x^{*}+y^{*}\right)^{2}} \\
& -a u \frac{\beta y^{*} g\left(v^{*}\right)}{\left(x^{*}+y^{*}\right)^{2}} \text {. }
\end{aligned}
$$

From the inequality of (12), we can conclude that all the terms of $\operatorname{det}\left(J\left(Q_{2}\right)\right)$ are negative, so $\operatorname{det}\left(J\left(Q_{2}\right)\right)<0$. This verifies assumption (4) of Lemma 5 . Hence, $Q_{2}$ is globally asymptotically stable if $R_{0}>1$ by Lemma 5 . This completes the proof. 


\section{Concluding Remarks}

In this paper, we consider a viral infection model with a general standard incidence rate. The basic reproduction number of model (2) is independent of the number of the host's organ prior to the infection, which avoids the emergence of the unreasonable situation for the basic viral models of Nowak and May and Perelson and Nelson. This general incidence rate represents a variety of possible incidences such as saturation response and standard incidence. The existence and uniqueness of the positive equilibrium of model (2) have been proved in this paper. We also show the positivity and boundedness of solutions of model (2). The global stability of the infection-free equilibrium and endemic equilibrium has been analyzed, respectively. When the basic reproduction number $R_{0}<1$, the infection-free equilibrium is globally asymptotically stable and the virus is cleared. Moreover, if $R_{0}>1$, then the endemic equilibrium is globally asymptotically stable and the virus persists in the host.

\section{Conflict of Interests}

The authors declare that there is no conflict of interests regarding the publication of this paper.

\section{Acknowledgments}

This research is jointly supported by the Research Foundation for Youth Scholars of Beijing Technology and Business University (QNJJ2013-24) and the General Program of Science and Technology Development Project of Beijing Municipal Education Commission (KM201310011006).

\section{References}

[1] P. de Leenheer and H. L. Smith, "Virus dynamics: a global analysis," SIAM Journal on Applied Mathematics, vol. 63, no. 4, pp. 1313-1327, 2003.

[2] S. Lewin, T. Walters, and S. Locarnini, "Hepatitis B treatment: rational combination chemotherapy based on viral kinetic and animal model studies," Antiviral Research, vol. 55, no. 3, pp. 381396, 2002.

[3] M. A. Nowak and R. M. May, Virus Dynamics, Oxford University Press, Oxford, UK, 2000.

[4] A. S. Perelson and P. W. Nelson, "Mathematical analysis of HIV1 dynamics in vivo," SIAM Review, vol. 41, no. 1, pp. 3-44, 1999.

[5] L. Min, Y. Su, and Y. Kuang, "Mathematical analysis of a basic virus infection model with application to HBV infection," The Rocky Mountain Journal of Mathematics, vol. 38, no. 5, pp. 15731585, 2008.

[6] S. A. Gourley, Y. Kuang, and J. D. Nagy, "Dynamics of a delay differential equation model of hepatitis B virus infection," Journal of Biological Dynamics, vol. 2, no. 2, pp. 140-153, 2008.

[7] T. Wang, Z. Hu, F. Liao, and W. Ma, "Global stability analysis for delayed virus infection model with general incidence rate and humoral immunity," Mathematics and Computers in Simulation, vol. 89, pp. 13-22, 2013.

[8] L. M. Cai and X. Z. Li, "Analysis of a SEIV epidemic model with a nonlinear incidence rate," Applied Mathematical Modelling, vol. 33, no. 7, pp. 2919-2926, 2009.
[9] K. Hattaf, N. Yousfi, and A. Tridane, "Mathematical analysis of a virus dynamics model with general incidence rate and cure rate," Nonlinear Analysis: Real World Applications, vol. 13, no. 4, pp. 1866-1872, 2012.

[10] Z. P. Wang and R. Xu, "Stability and Hopf bifurcation in a viral infection model with nonlinear incidence rate and delayed immune response," Communications in Nonlinear Science and Numerical Simulation, vol. 17, no. 2, pp. 964-978, 2012.

[11] K. Wang, A. Fan, and A. Torres, "Global properties of an improved hepatitis B virus model," Nonlinear Analysis. Real World Applications, vol. 11, no. 4, pp. 3131-3138, 2010.

[12] X. Song and A. U. Neumann, "Global stability and periodic solution of the viral dynamics," Journal of Mathematical Analysis and Applications, vol. 329, no. 1, pp. 281-297, 2007.

[13] Z. X. Hu, J. J. Zhang, H. Wang, W. Ma, and F. Liao, "Dynamics analysis of a delayed viral infection model with logistic growth and immune impairment," Applied Mathematical Modelling, vol. 38, no. 2, pp. 524-534, 2014.

[14] J. A. Yorke and W. P. London, "Recurrent outbreaks of measles, chickenpox and mumps. II. Systematic differences in contact rates and stochastic effects," The American Journal of Epidemiology, vol. 98, no. 6, pp. 469-482, 1973.

[15] W. M. Liu, H. W. Hethcote, and S. A. Levin, "Dynamical behavior of epidemiological models with nonlinear incidence rates," Journal of Mathematical Biology, vol. 25, no. 4, pp. 359380, 1987.

[16] M. Y. Li and L. C. Wang, "Global stability in some SEIR epidemic models," in Mathematical Approaches for Emerging and Reemerging Infectious Diseases: Models, Methods, and Theory, vol. 126 of The IMA Volumes in Mathematics and Its Applications, pp. 295-311, Springer, New York, NY, USA, 2002.

[17] G. Butler and P. Waltman, "Persistence in dynamical systems," Journal of Differential Equations, vol. 63, no. 2, pp. 255-263, 1986.

[18] M. Y. Li, J. R. Graef, L. Wang, and J. Karsai, "Global dynamics of a SEIR model with varying total population size," Mathematical Biosciences, vol. 160, no. 2, pp. 191-213, 1999. 


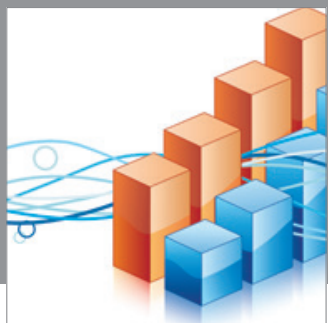

Advances in

Operations Research

mansans

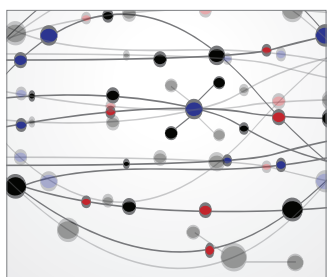

The Scientific World Journal
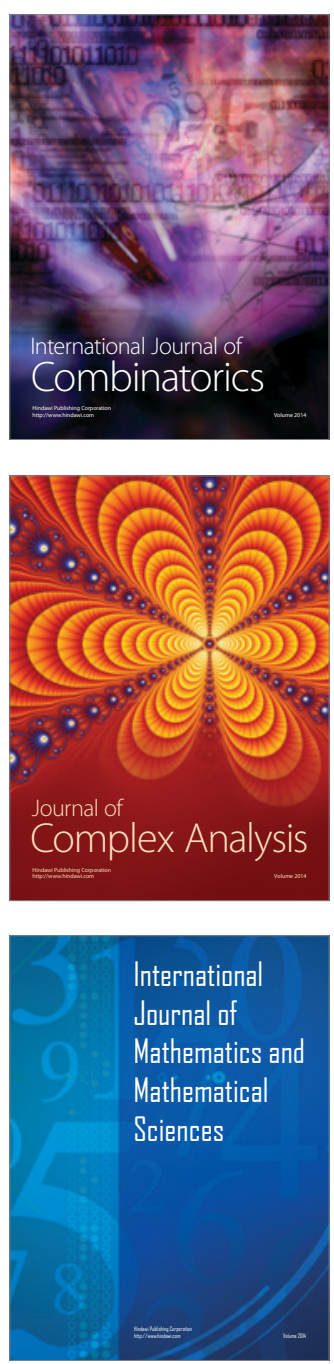
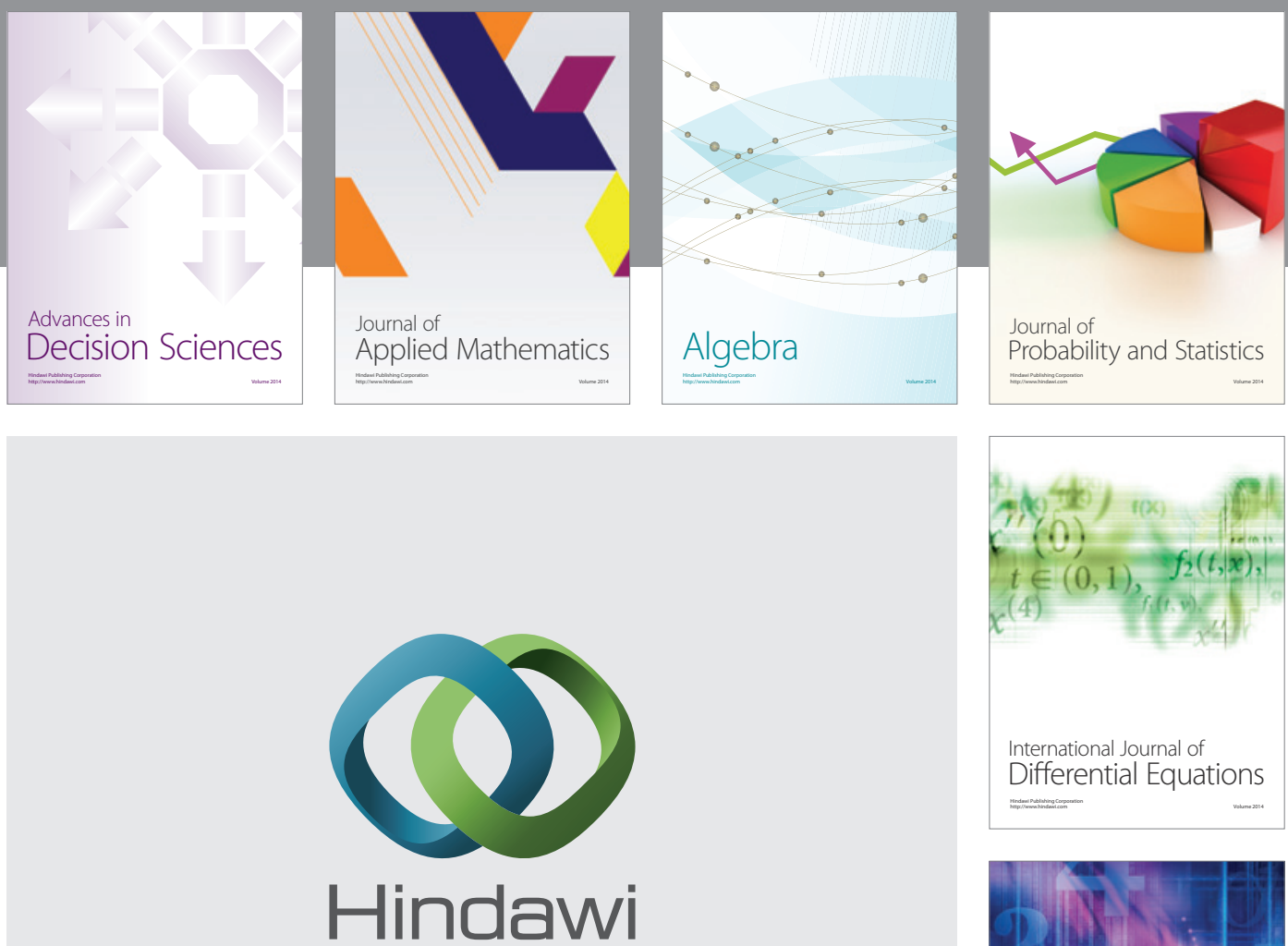

Submit your manuscripts at http://www.hindawi.com
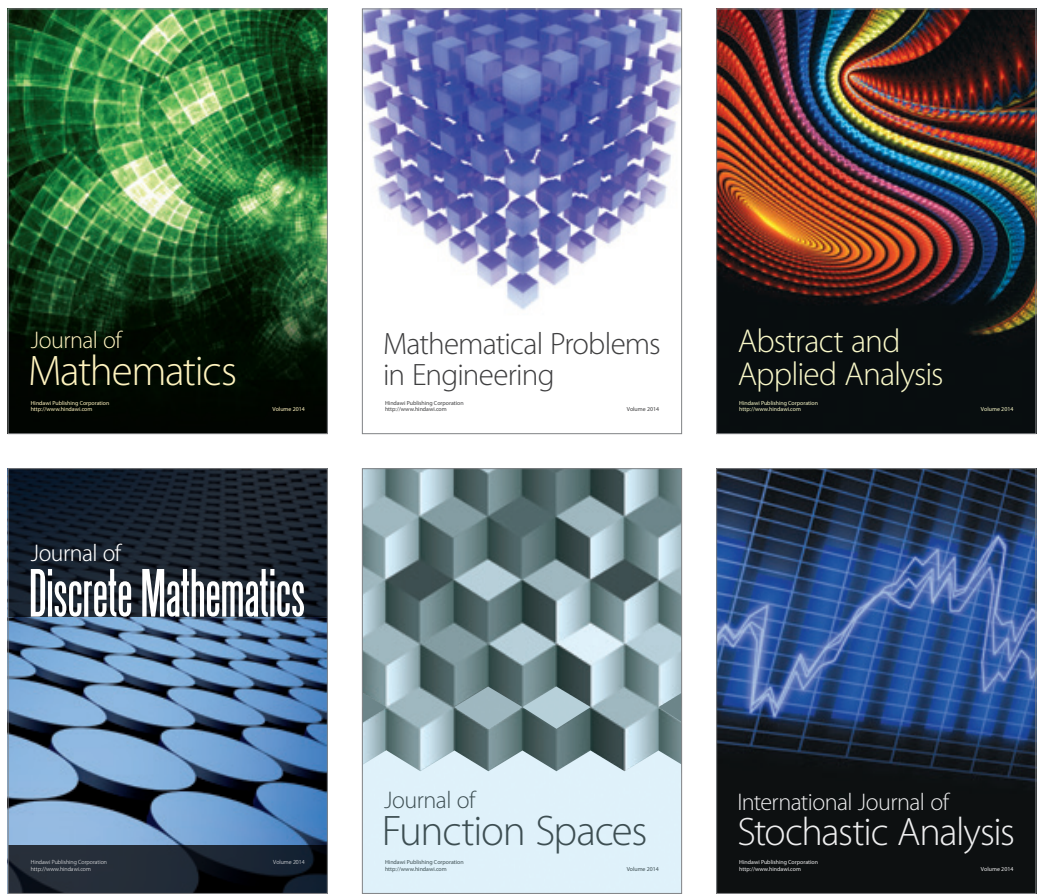

Journal of

Function Spaces

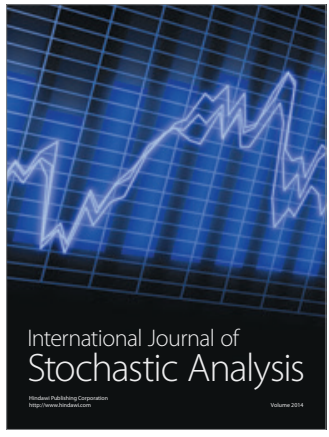

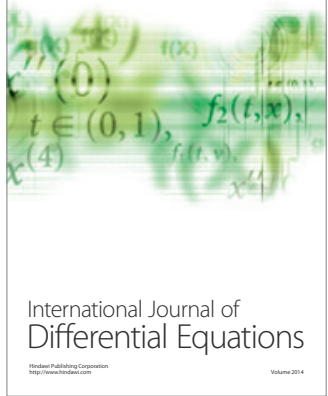
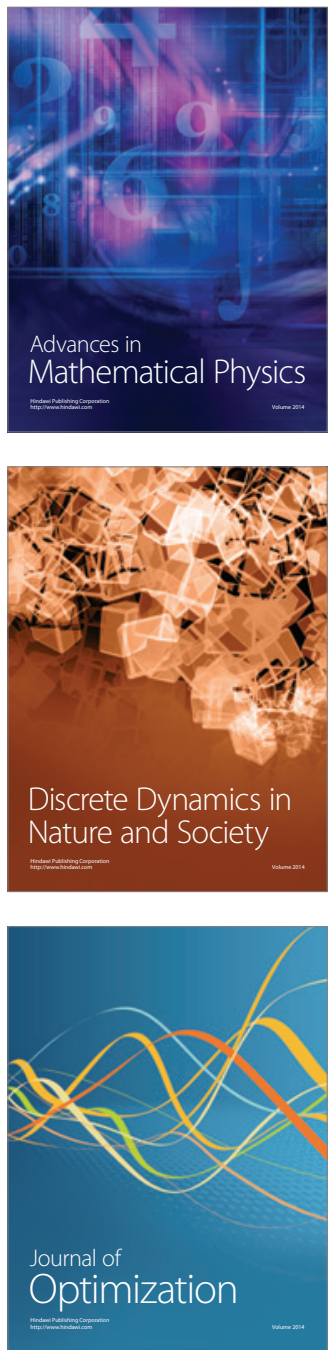